\title{
Nucleus accumbens-1/GADD45GIP1 axis mediates cisplatin resistance through cellular senescence in ovarian cancer
}

\author{
KENTARO NAKAYAMA $^{1 *}$, MUNMUN RAHMAN $^{1 *}$, MOHAMMED TANJIMUR RAHMAN $^{1}$, \\ KOHEI NAKAMURA ${ }^{1}$, EMI SATO ${ }^{1}$, HIROSHI KATAGIRI ${ }^{1}$, TOMOKA ISHIBASHI ${ }^{1}$, MASAKO ISHIKAWA ${ }^{1}$, \\ KOUJI IIDA ${ }^{1}$, SULTANA RAZIA ${ }^{1}$, NORIYUKI ISHIKAWA ${ }^{2}$ and SATORU KYO ${ }^{1}$ \\ Departments of ${ }^{1}$ Obstetrics and Gynecology, and ${ }^{2}$ Organ Pathology, \\ Shimane University School of Medicine, Izumo, Shimane 6938501, Japan
}

Received September 23, 2015; Accepted January 4, 2017

DOI: $10.3892 / 01.2017 .6099$

\begin{abstract}
Nucleus accumbens-1 (NAC1), a nuclear factor belonging to the bric-a-brac-tramtrack-broad complex/pox virus and zinc finger gene family, is known to serve important roles in the proliferation and growth of tumor cells, and in chemotherapy resistance. However, the underlying molecular mechanisms through which NAC1 contributes to drug resistance remain unclear. In the present study, the role of NAC1 in drug resistance in ovarian cancer was investigated. NAC1 expression was markedly negatively associated with growth arrest and DNA-damage-inducible $45 \gamma$-interacting protein 1 (GADD45GIP1) expression in ovarian cancer. Increased NAC1 expression or decreased GADD45GIP1 expression was significantly associated with decreased progression-free survival $(\mathrm{P}=0.0041)$. Multivariate analysis demonstrated that NAC1/GADD45GIP1 expression was an independent prognostic factor of progression-free survival $(\mathrm{P}=0.0405)$. It was investigated whether cellular senescence was involved in NAC1-mediated resistance to cisplatin, a commonly used chemotherapeutic drug in the treatment of ovarian cancer. Treatment with cisplatin activated cellular senescence in ovarian cancer cell lines (SKOV3 and TOV-21G cells). Furthermore, knockdown of NAC1 by RNA interference significantly increased GADD45GIP1 expression and inhibited cisplatin-induced cellular senescence, resulting in increased cisplatin cytotoxicity in SKOV3 cells, which express increased
\end{abstract}

Correspondence to: Dr Kentaro Nakayama, Department of Obstetrics and Gynecology, Shimane University School of Medicine, Enyacho 89-1, Izumo, Shimane 6938501, Japan

E-mail:kn88@med.shimane-u.ac.jp

${ }^{*}$ Contributed equally

Abbreviations: BTB, bric-a-brac-tramtrack-broad complex; CDDP, cisplatin

Key words: ovarian carcinoma, nucleus accumbens-1, growth arrest and DNA-damage-inducible $\gamma$-interacting protein 1 , senescence, chemotherapy resistance, cisplatin levels of NAC1. To investigate whether the sensitizing effect of NAC1 inhibition on cisplatin-induced cytotoxicity may be attributed to the suppression of cellular senescence, the effects of NAC1 overexpression were assessed in TOV-21G cells, which do not express endogenous NAC1. Transfection with NAC1 in TOV-21G cells reduced the sensitivity of TOV-21G cells to cisplatin, indicating that suppression of cellular senescence was induced by GADD45GP1 activation. The results of the present study suggest that $\mathrm{NAC} 1$ is a negative regulator of cellular senescence and that NAC1-dependent suppression of senescence, mediated through GADD45GIP1, serves an important role in promoting cisplatin resistance. Therefore, the NAC1/GADD45GIP1 axis may be a potential target for the treatment of ovarian cancer, particularly in platinum-resistant cancers.

\section{Introduction}

Ovarian cancer is the leading cause of mortality in women with gynecological cancer (1). Advanced stage cancer, which is associated with increased morbidity and mortality, is diagnosed in $>70 \%$ of patients with ovarian cancer (2). In spite of initial responsiveness to conventional chemotherapy with platinum- and taxane-based drugs, the majority of patients develop chemoresistant tumors and succumb to the disease (2). Therefore, treatment failure is often attributed to primary or acquired resistance to chemotherapeutic agents, representing a considerable problem in the management of the majority of patients with cancer (2).

In a previous study, the present authors characterized nucleus accumbens-1 (NAC1) as a candidate protein involved in chemoresistance in ovarian cancer (3). NAC1 is a nuclear protein belonging to the bric-a-brac-tramtrack-broad complex/pox virus and zinc finger domain family (4). In ovarian carcinomas, NAC1 expression is markedly increased in recurrent tumors following chemotherapeutic intervention compared with that in primary tumors prior to treatment $(3,4)$. Upregulation of NAC1 contributes to tumor cell growth, survival, migration, invasion and resistance to chemotherapeutic drugs (3-6). Previously, the present authors demonstrated that $\mathrm{NAC} 1$ protein negatively regulates the expression of growth arrest and DNA damage-inducible 
45 $\gamma$ (GADD45G) (4) and GADD45G-interacting protein 1 (GADD45GIP1) (6). GADD45GIP1 has been demonstrated to interact with all isoforms of GADD45; this interaction enhances the functions of the GADD45 complex (7). NAC1 contributes to tumor growth and survival by inhibiting GADD45GIP1 expression (6). Furthermore, NAC1 has been demonstrated to contribute to chemoresistance to paclitaxel and carboplatin in ovarian cancer through the inactivation of the GADD45 pathway (4). However, the specific functions of $\mathrm{NAC1}$ in tumor development, recurrence and chemoresistance remain unclear.

$\mathrm{NAC} 1$ has been identified as a negative regulator of cellular senescence (8). Furthermore, suppression of senescence by $\mathrm{NAC1}$ serves an important role in promoting tumorigenesis and improved treatment outcomes (8). Cellular senescence is defined as the irreversible growth arrest of cells in the $\mathrm{G}_{1}$-phase of the cell cycle, and is frequently characterized by flattened and enlarged cell morphology, increased cytoplasmic granularity, and elevated activity of senescence-associated $\beta$-galactosidase (SA $\beta$ gal) (8-10). Senescence may occur following a number of cell divisions or be induced by various stimuli, including DNA damage, oncogene activation, telomere shortening, and treatment with DNA-damaging drugs or irradiation $(10,11)$. Furthermore, senescent cells require decreased doses of chemotherapeutic drugs to induce cell death compared with those required to drive non-senescent cells into apoptosis. This may substantially improve anticancer strategies and reduce the side effects of many treatment procedures (10-12). Therefore, therapy-induced senescence may influence the outcome of treatments, whereas evasion of senescence may induce tumorigenesis, cancer recurrence and treatment failure.

In the present study, the role of $\mathrm{NACl}$ in ovarian cancer was investigated by examining the association between NAC1 and GADD45GIP1 protein expression in tissue samples from patients with ovarian carcinoma, and evaluating the prognostic significance of NAC1/GADD45GIP1 expression. The underlying molecular mechanisms of NAC1-mediated senescence through GADD45GIP1 in ovarian cancer cells were also investigated.

\section{Materials and methods}

Tissue samples. A total of 49 paraffin-embedded tumor tissues from female patients with advanced (stages III or IV) ovarian cancer were obtained from the Department of Obstetrics and Gynecology at Shimane University Hospital (Izumo, Japan), all of whom underwent surgery at Shimane University Hosipital between January 1998 and December 2008. The 49 patients with ovarian cancer were aged from 46 to 76 years (median, 61 years). All tissue specimens were collected after obtaining written consent from patients with the approval of the Facility Ethical Committee (Shimane University Hospital; approval no. 2004-0381). Diagnosis was based on the conventional morphological examination of sections stained with hematoxylin and eosin (H\&E), and tumors were classified according to the World Health Organization classification (13). All patients were primarily treated with cytoreductive surgery, and adjuvant platinum and taxane chemotherapy $(5 \mathrm{mg} / \mathrm{ml} \mathrm{x}$ min carboplatin with
$175 \mathrm{mg} / \mathrm{m}^{2}$ paclitaxel or $70 \mathrm{mg} / \mathrm{m}^{2}$ docetaxel). All patients received between 6 and 12 courses of this regimen. The acquisition of tumor tissues was approved by the Shimane University Institutional Review Board (Izumo, Japan). The paraffin tissue blocks were organized into tissue microarrays, each made by removing cores ( $3 \mathrm{~mm}$ in diameter) of tumor tissues from the block. Selection of the area to core was made by a gynecological oncologist and pathology technician, and was based on a review of the $\mathrm{H} \& \mathrm{E}$ slides.

Immunohistochemistry. Briefly, tissue sections were dewaxed in xylene for $10 \mathrm{~min}$ at $20^{\circ} \mathrm{C}$, rehydrated in graded ethanol, washed in phosphate-buffered solution ( $\mathrm{pH}$ 7.25) for $5 \mathrm{~min}$ and quenched in peroxidase-blocking reagent for $5 \mathrm{~min}$ at $20^{\circ} \mathrm{C}$ to remove endogenous peroxidase activity. Following antigen retrieval in sodium citrate buffer ( $\mathrm{pH}$ 7.0), slides were incubated overnight at $4^{\circ} \mathrm{C}$ with mouse monoclonal anti-NAC1 antibody (cat. no. NB110-77345; Novus Biologicals, LLC, Littleton, CO, USA) and mouse monoclonal anti-GADD45GIP1 antibodies (cat. no. LS-C120010; LifeSpan BioSciences, Inc., Seattle, WA, USA) at a dilution of 1:100 followed by detection using the peroxidase method with the EnVision+ System (Dako; Agilent Technologies, Inc., Santa Clara, CA, USA) according to the manufacturer's protocol. Immunohistochemical signal intensity was scored by two investigators using a four-tier system: 0 , undetectable; $1+$, weakly positive; $2+$, moderately positive; and $3+$, intensely positive (2). Scores of 0 and $1+$ indicated negative results, whereas scores of $2+$ and $3+$ were regarded as positive results.

Cell lines and cell culture. The SKOV3 (serous carcinoma) and TOV-21G (clear cell carcinoma) human ovarian carcinoma cell lines were obtained from the American Type Culture Collection (Manassas, VA, USA). All cell lines were maintained in Dulbecco's modified Eagle's medium (Thermo Fisher Scientific, Inc., Waltham, MA, USA) supplemented with $10 \%$ fetal bovine serum, $100 \mathrm{U} / \mathrm{ml}$ penicillin and $100 \mu \mathrm{g} / \mathrm{ml}$ streptomycin. All cells were seeded into Cellstars ${ }^{\circledR}$ tissue culture plates (Greiner Bio-One $\mathrm{GmbH}$, Frickenhausen, Germany) in a humidified incubator containing $5 \% \mathrm{CO}_{2}$ at $37^{\circ} \mathrm{C}$.

Transfection with NACl small interfering RNA (siRNA). Two siRNAs targeting NAC1 were designed with the following sense sequences: 5'-UGAUGUACACGUUGGUGCCUGUCA CCA-3' and 5'-GAGGAAGAACUCGGUGCCCUUCUC CAU-3'. Control siRNA (luciferase siRNA) was purchased from Invitogen (cat. no. 12935-146; Thermo Fisher Scientific, Inc.). A total of 5,000 SKOV3 cells/well were seeded in 96-well plates and transfected with siRNAs using Oligofectamine ${ }^{\text {Tx }}$ (Invitrogen; Thermo Fisher Scientific, Inc.) according to the manufacturer's protocol.

Retroviral transfection and generation of NACl. The TOV-21G cells were used for transfection when the confluence reached $70 \%$. The NAC1 retroviral vector (pWZL-Hygro retroviral vector) was donated by Dr Ie-Ming Shih (Johns Hopkins Medical Institutions, Baltimore, MD, USA). Packaging cells (Phoenix cells; Invitrogen; Thermo Fisher Scientific, Inc.) were transiently transfected with the NAC1 construct or empty 
vector using Lipofectamine ${ }^{\mathrm{TM}}$ (Invitrogen; Thermo Fisher Scientific, Inc.) according to the manufacturer's protocol. The following day, the supernatant was harvested and passed through a $0.45 \mu \mathrm{m}$ syringe filter. The filtered viral supernatant was resuspended in $4 \mu \mathrm{g} / \mathrm{ml}$ polybrene (Sigma-Aldrich; Merck KGaA, Darmstadt, Germany) and added to the TOV-21G ovarian cancer cell cultures. Cells were incubated for $24 \mathrm{~h}$ following infection at $37^{\circ} \mathrm{C}$ and subsequently harvested using Trypsin-EDTA for use in assays.

SA $\beta$ gal assay. A total of 5,000 TOV-21G and SKOV3 cells were seeded into 96 -well plates. Following appropriate exposure to 1,2 or $5 \mu \mathrm{M}$ cisplatin, cells at $37^{\circ} \mathrm{C}$ for $24 \mathrm{~h}$. Cells were stained for $\beta$-galactosidase activity as previously described by Dimri et al (9). Cells were washed twice with PBS, fixed with $2 \%$ formaldehyde and $0.2 \%$ glutaraldehyde at room temperature for $1 \mathrm{~h}$, washed three times with PBS, and incubated at $37^{\circ} \mathrm{C}$ overnight in $\mathrm{X}$-gal staining solution using a Senescent $\beta$-Galactosidase Staining kit (Sigma-Aldrich; Merck KGaA) according to the manufacturer's protocol. Blue-stained senescent cells were counted using a light microscope. Cell morphology was analyzed using a light microscope.

Western blot analysis. Cell lysates were prepared from siRNA-transfected SKOV3 cells and NAC1-transfected TOV-21G cells following exposure to 1,2 or $5 \mu \mathrm{M}$ cisplatin at $37^{\circ} \mathrm{C}$ for $24 \mathrm{~h}$. Equal amounts of total protein $(20 \mu \mathrm{g} / \mathrm{well})$ from each lysate were separated on $10 \%$ Tris-glycineSDS-polyacrylamide gels (Novex; Thermo Fisher Scientific, Inc.) using SDS/PAGE and transferred using electroblotting onto Immobilon-P polyvinylidene difluoride membranes (EMD Millipore, Billerica, MA, USA). Membranes were probed overnight at $4^{\circ} \mathrm{C}$ with anti-NAC1 antibodies (cat. no. NB110-77345; 1:1,000 dilution; Novus Biologicals, Littleton, CO, USA) and anti-GADD45GIP1 antibodies (cat. no. LS-C120010; 1:500 dilution; LifeSpan Biosciences, Inc.) followed by incubation with horseradish peroxidase-conjugated anti-mouse immunoglobulin (cat. no. 715-035-1500; 1:10,000 dilution; Jackson ImmunoReasearch Laboratories, Inc., West Grove, PA, USA) at room temperature for $1 \mathrm{~h}$. The same membranes were probed with anti-GAPDH antibodies overnight at $4^{\circ} \mathrm{C}$ (cat no. 5174; 1:10,000 dilution; Cell Signaling Technology, Inc., Danvers, MA, USA) as a loading control. Western blots were developed by chemiluminescence, according to the manufacturer's protocol (cat. no. 62242; Pierce; Thermo Fisher Scientific, Inc.).

Statistical analysis. Statistical analyses were conducted using SPSS software (version 19.0; IBM Corp., Armonk, NY, USA). Results are presented as the mean \pm standard deviation from triplicate determinations. Progression-free and overall survival rates were calculated between the date of diagnosis and the date of first relapse or last follow-up. Survival data were plotted as Kaplan-Meier estimator curves, and the statistical significance was determined using the log-rank test. Data were censored when patients were lost to follow-up. The Student's $t$ test was used to analyze the significance of differences. $\mathrm{P}<0.05$ was considered to indicate a statistically significant difference.
Table I. Association between NAC1 and GADD45GIP1 expression.

\begin{tabular}{lccc}
\hline & \multicolumn{3}{c}{ NAC1 expression } \\
\cline { 2 - 4 } GADD45GIP1 expression & Increased & Decreased & P-value \\
\hline Increased & 0 & 13 & $\mathrm{P}<0.05$ \\
Decreased & 12 & 24 & \\
\hline
\end{tabular}

NAC1, nucleus accumbens-1; GADD45GIP1, growth arrest and DNA-damage-inducible $45 \gamma$-interacting protein 1.

\section{Results}

Expression of NAC1 and GADD45GIPl protein is inversely associated in ovarian carcinoma. Among 49 ovarian carcinomas, increased NAC1 expression (immunohistochemical intensity of $2+$ or $3+$ ) was observed in 12 cases (24\%), and increased GADD45GIP1 expression was identified in 13 cases $(26 \%)$. NAC1 immunoreactivity was determined in tumor cell nuclei (Fig. 1A and B). GADD45GIP1 immunoreactivity was detected in the cytoplasm (Fig. 1C and D). All 12 cases with increased NAC1 expression exhibited decreased GADD45GIP1 expression (Table I), whereas the remaining 37 cases with decreased NAC1 expression exhibited decreased (24 cases) or increased (13 cases) GADD45GIP1 expression. Increased NAC1 expression was significantly inversely associated with decreased GADD45GIP1 expression in ovarian carcinomas $(\mathrm{P}<0.05)$.

Increased NACl and decreased GADD45GIP1 protein expression are associated with decreased overall/progression-free survival. Of the 49 ovarian carcinoma samples examined in the present study, 45 were used for clinicopathological and prognostic analysis. Using immunohistochemical analysis, NAC1 and GADD45GIP1 were identified to be markedly negatively associated with each other. When patients with ovarian carcinomas treated with platinum-based chemotherapy were classified using a two-tier system based on expression level (decreased or increased), patients with increased NAC1 expression or decreased GADD45GIP1 expression exhibited significantly decreased progression-free survival compared with patients exhibiting decreased NAC1 expression or increased GADD45GIP1 expression ( $\mathrm{P}=0.0411$, log-rank test; Fig. 2A). The presence of increased NAC1 expression or decreased GADD45GIP1 expression tended to be associated with decreased overall survival compared with decreased NAC1 expression or increased GADD45GIP1 expression; however, this was not identified to be significant $(\mathrm{P}=0.0711$; Table II; Fig. 2B). When the data were stratified using multivariate analysis, either increased NAC1 expression or decreased GADD45GIP1 expression and residual tumor $(\geq 1 \mathrm{~cm})$ remained a significant predictor for decreased progression-free survival ( $\mathrm{P}=0.0405$ and 0.0457 , respectively; Table III).

NACl suppresses therapy-induced cellular senescence in ovarian cancer cells. A previous study demonstrated that 

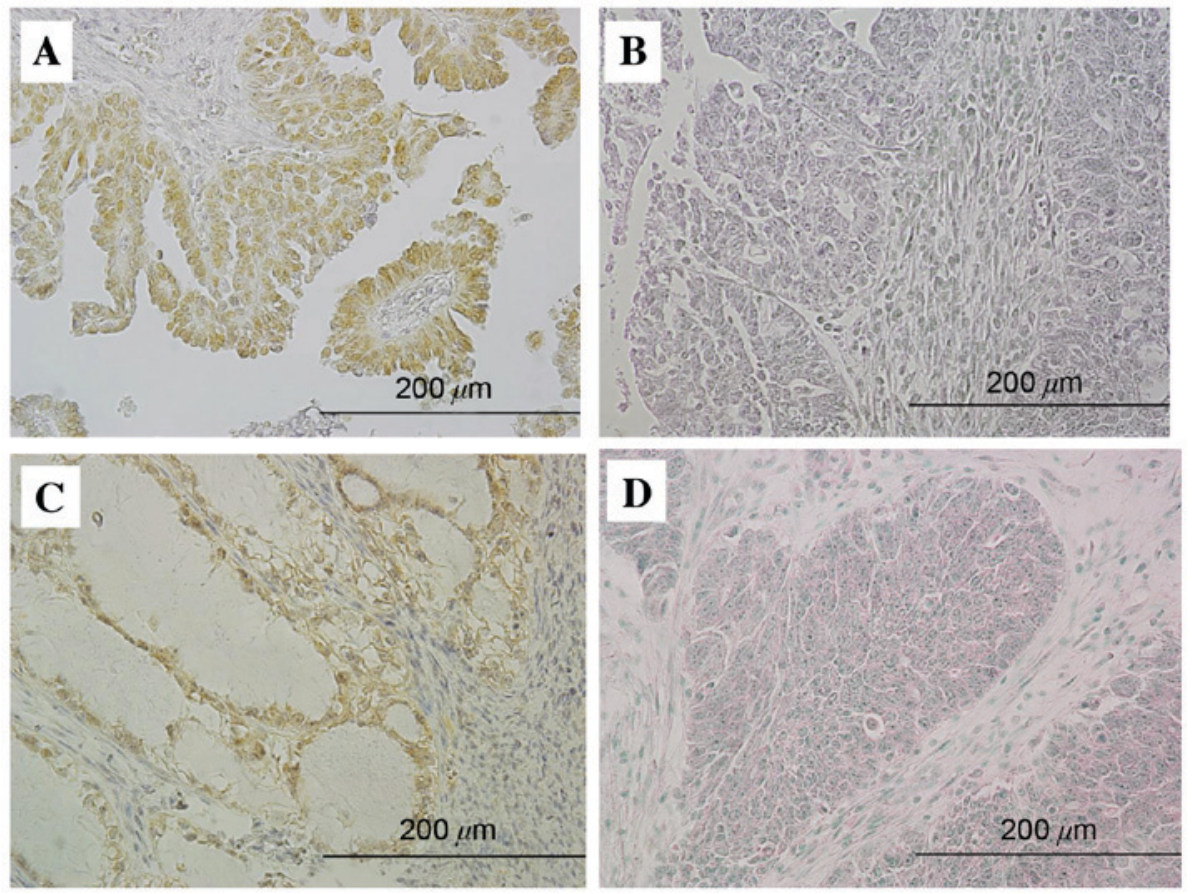

Figure 1. Immunoreactivity of NAC1 and GADD45GIP1 in ovarian cancer tissues. Representative images of (A) intense NAC1 immunoreactivity in the nuclei of ovarian cancer cells, (B) negative NAC1 staining in ovarian cancer tissues, (C) intense GADD45GIP1 immunoreactivity in the cytoplasm of ovarian cancer cells, and (D) negative GADD45GIP1 staining in ovarian cancer tissue. NAC1, nucleus accumbens-1; GADD45GIP1, growth arrest and DNA-damage-inducible $45 \gamma$-interacting protein 1 .
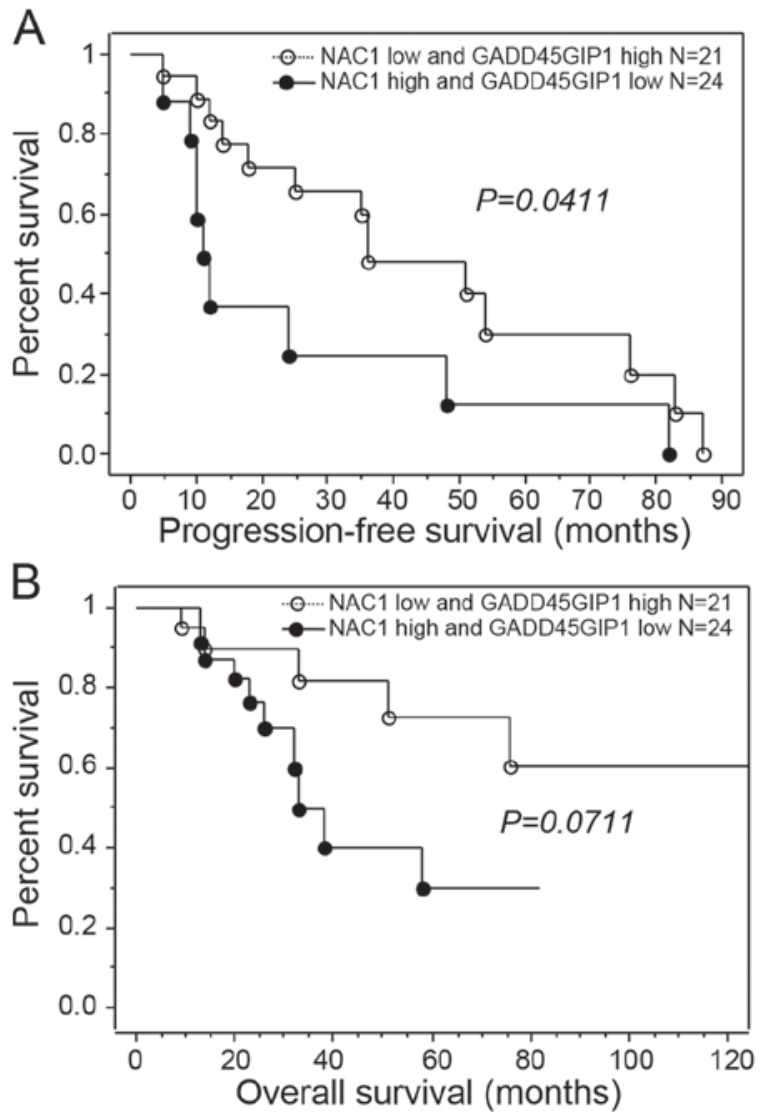

Figure 2. Increased NAC1 or decreased GADD45GIP1 expression is (A) significantly associated with decreased progression-free survival $(\mathrm{P}=0.0411)$, and (B) tends to be associated with decreased overall survival $(\mathrm{P}=0.0711)$ in patients with ovarian carcinoma treated with platinum-based chemotherapy. NAC1, nucleus accumbens-1; GADD45GIP1, growth arrest and DNA-damage-inducible $45 \gamma$-interacting protein 1 .
NAC1 negatively regulates senescence in tumor cells (8). In the present study, this phenomenon was investigated further following treatment of ovarian cancer cells with cisplatin, the standard chemotherapeutic drug used for ovarian cancer treatment. Various sublethal doses of cisplatin $(1,2$ and $5 \mu \mathrm{M})$ were used to induce senescence. Sublethal doses of cisplatin significantly increased the number of blue-stained senescent cells $(\mathrm{P}<0.05$; Fig. 3A and $\mathrm{B})$, and the number of cells with flattened and enlarged morphology compared with that in untreated cells (data not shown).

As presented in Fig. 3A, NAC1 transfection of cisplatin-treated TOV-21G cells caused a significant decrease in SA $\beta$ gal staining compared with that in untransfected cisplatin-treated cells. To confirm this result, the SA $\beta$ gal assay was repeated using NAC1 siRNA transfection in SKOV3 cells, which have higher endogenous NAC1 expression. As presented in Fig. 3B, NAC1 knockdown in cisplatin-treated SKOV3 cells caused a significant increase in the number of Saßgal-positive cells compared with that in control siRNA-transfected SKOV3 cells $(\mathrm{P}<0.05)$. These results further confirmed that NAC1 inactivates stress-induced cellular senescence following therapeutic intervention.

NACl prevents therapy-induced senescence possibly through inactivation of GADD45GIP1. The gene coding for GADD45GIP1 is a downstream target regulated by NAC1 (6), and acts as a negative regulatory factor for cell cycle progression and cell growth (7). Therefore, it was hypothesized that GADD45GIP1 contributes to the NAC1-mediated suppression of cellular senescence. It was identified that NAC1-transfected TOV-21G cells exhibited decreased GADD45GIP1 protein expression, whereas NAC1-deficient TOV-21G cells exhibited 
Table II. Univariate analysis of overall prognostic factors in patients with ovarian cancer.

\begin{tabular}{|c|c|c|c|c|}
\hline \multirow[b]{2}{*}{ Factor } & \multirow[b]{2}{*}{$\mathrm{n}$} & \multicolumn{3}{|c|}{ Univariate analysis } \\
\hline & & Hazard ratio & $95 \% \mathrm{CI}$ & P-value \\
\hline FIGO stage & & 2.8 & $0.4-21.7$ & 0.3154 \\
\hline $\mathrm{I} / \mathrm{II}$ & 10 & & & \\
\hline III/IV & 35 & & & \\
\hline Histology & & 1 & $0.3-3.6$ & 0.9903 \\
\hline Serous & 33 & & & \\
\hline Others & 12 & & & \\
\hline Age, years & & 0.9 & $0.3-3.0$ & 0.9169 \\
\hline$<60$ & 12 & & & \\
\hline$\geq 60$ & 33 & & & \\
\hline Residual tumor size, $\mathrm{cm}$ & & 3.9 & $0.9-17.7$ & 0.08 \\
\hline$<1$ & 9 & & & \\
\hline$\geq 1$ & 36 & & & \\
\hline NAC1/GADD45GIP1 status & & 2.8 & $0.9-8.4$ & 0.0711 \\
\hline Increased NAC1 or decreased GADD45GIP1 & 24 & & & \\
\hline Others & 21 & & & \\
\hline
\end{tabular}

CI, confidence interval; FIGO, international federation of gynecology and obstetrics; NAC1, nucleus accumbens-1; GADD45GIP1, growth arrest and DNA-damage-inducible $45 \gamma$-interacting protein 1.

Table III. Univariate and multivariate analysis of progression-free prognostic factors in patients with ovarian cancer.

\begin{tabular}{|c|c|c|c|c|c|c|c|}
\hline \multirow[b]{2}{*}{ Factor } & \multirow[b]{2}{*}{$\mathrm{n}$} & \multicolumn{3}{|c|}{ Univariate analysis } & \multicolumn{3}{|c|}{ Multivariate analysis } \\
\hline & & Hazard ratio & $95 \% \mathrm{CI}$ & P-value & Hazard ratio & $95 \% \mathrm{CI}$ & P-value \\
\hline FIGO stage & & 1 & $0.3-2.9$ & 0.9389 & NA & NA & NA \\
\hline III & 10 & & & & & & \\
\hline IV & 35 & & & & & & \\
\hline Histology & & 1.6 & $0.6-3.8$ & 0.3185 & NA & NA & NA \\
\hline Serous & 33 & & & & & & \\
\hline Others & 12 & & & & & & \\
\hline Age, years & & 0.8 & $0.3-1.8$ & 0.5532 & NA & NA & NA \\
\hline$<60$ & 12 & & & & & & \\
\hline$\geq 60$ & 33 & & & & & & \\
\hline Residual tumor size, $\mathrm{cm}$ & & 2.8 & $1.0-7.9$ & 0.0457 & 2.9 & $1.0-8.6$ & 0.0514 \\
\hline$<1$ & 9 & & & & & & \\
\hline$\geq 1$ & 36 & & & & & & \\
\hline NAC1/GADD45GIP1 status & & 2.5 & $1.0-5.9$ & 0.0411 & 2.5 & $1.0-6.2$ & 0.0405 \\
\hline Increased NAC1 or decreased GADD45GIP1 & 24 & & & & & & \\
\hline Others & 21 & & & & & & \\
\hline
\end{tabular}

CI, confidence interval; FIGO, international federation of gynecology and obstetrics; NAC1, nucleus accumbens-1; GADD45GIP1, growth arrest and DNA-damage-inducible $45 \gamma$-interacting protein 1 .

increased GADD45GIP1 protein expression following treatment with the same sublethal doses of cisplatin that were used in the SA $\beta$ gal assay (Fig. 3C). Similarly, knockdown of
NAC1 in SKOV3 cells followed by treatment with cisplatin enhanced GADD45GIP1 expression compared with that in NAC1-overexpressing SKOV3 cells (Fig. 3D). 

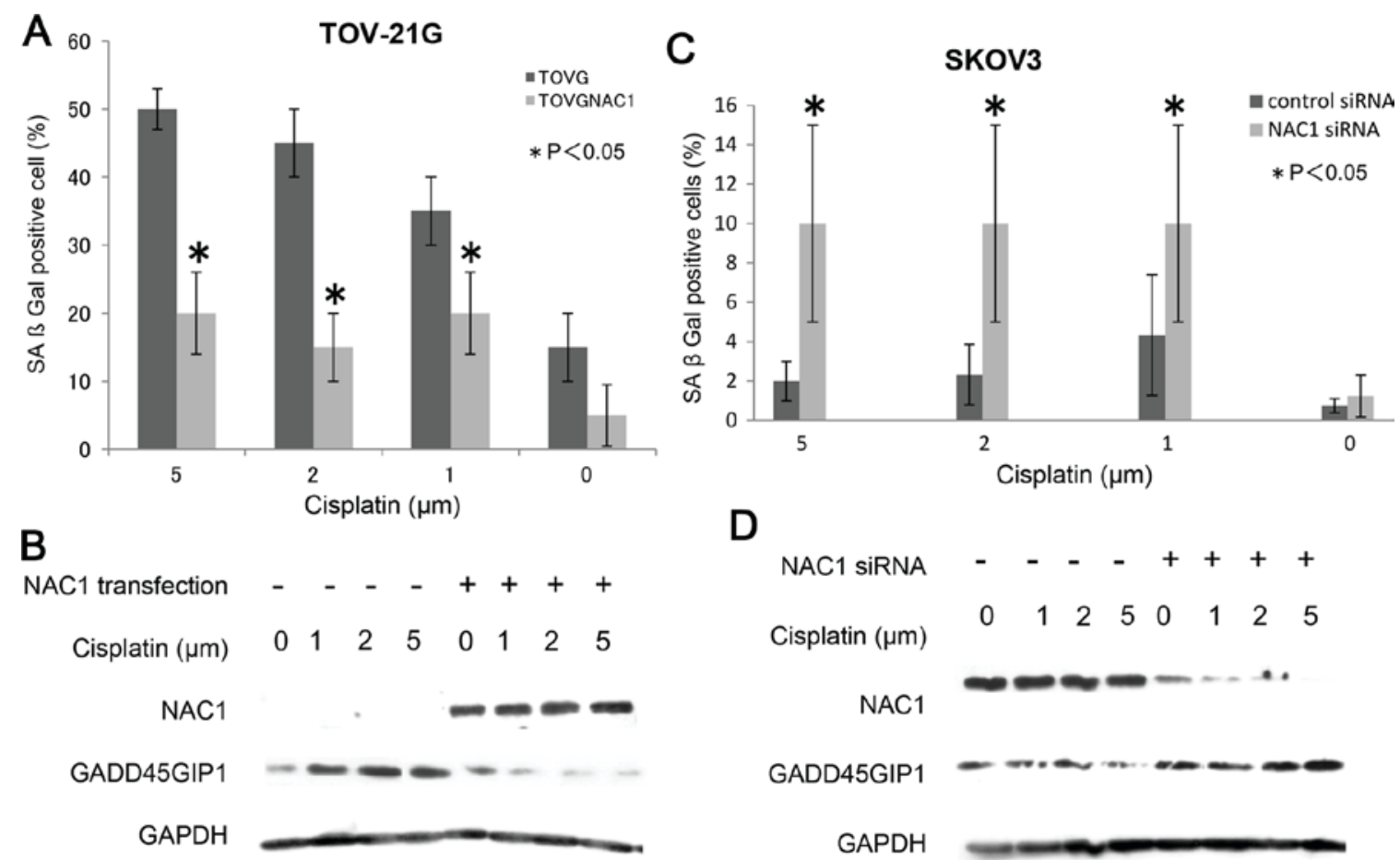

Figure 3. NAC1 expression increases Saßgal-positive cells and decreases GADD45GIP1 expression in ovarian cancer cells. (A) The number of SAßgal-positive TOV-21G cells transfected with an NAC1 construct followed by cisplatin treatment was significantly decreased compared with control vector-transfected TOV-21G cells treated similarly. Asterisks indicated TOV-21G NAC1 cells significantly decreased SAßgal-positive cells compared to TOV-21G cells. (B) Western blot analysis demonstrated that NAC1 transfection followed by cisplatin treatment decreased GADD45GIP1 expression in TOV-21G cells compared with untransfected TOV-21G cells. (C) The number of SAßgal-positive SKOV3 cells transfected with NAC1 siRNA followed by cisplatin treatment was significantly increased compared with control siRNA-transfected cells treated similarly. Asterisks indicated SKOV3 with NAC1 siRNA cells significantly increase SABgal-positive cells compared to SKOV3 with control siRNA cells. (D) Western blot analysis demonstrated that NAC1 siRNA followed by cisplatin treatment increased GADD45GIP1 expression in SKOV3 cells compared with untransfected SKOV3 cells. NAC1, nucleus accumbens-1; GADD45GIP1, growth arrest and DNA-damage-inducible $45 \gamma$-interacting protein 1; siRNA, small interfering RNA.

\section{Discussion}

The majority of patients with ovarian cancer are initially responsive to carboplatin-paclitaxel combination chemotherapy; however, the majority of patients eventually develop recurrent chemoresistant tumors, contributing to increased mortality rates in patients with ovarian cancer (2). In a previous study, the authors demonstrated that NAC1 upregulation in ovarian serous carcinomas was significantly associated with early tumor recurrence following cytoreduction therapy and carboplatin-paclitaxel combined chemotherapy (3).

In the present study, a previously unrecognized role for $\mathrm{NAC} 1$ in regulating cellular senescence was identified, which was demonstrated to result in cisplatin resistance. Furthermore, a potential underlying molecular mechanism was investigated for the contribution of NAC1 upregulation, as observed in ovarian cancer, to early recurrence in patients following chemotherapy, as previously reported $(2,14)$. Treatment with the chemotherapeutic drug cisplatin was demonstrated to activate cellular senescence in ovarian cancer cells, and inactivation and gene silencing of NAC1 inhibited the activation of cellular senescence by cisplatin. It was further demonstrated that regulation of cellular senescence by NAC1 was mediated through its effects on GADD45GIP1. Previously, the present authors demonstrated that NAC1 negatively regulates the expression of GADD45GIP1 (6) and GADD45G (4). Additionally, Zhang et al (15) recently demonstrated that
GADD45G promotes cellular senescence in hepatocellular carcinoma (HCC) cells and markedly suppresses tumor growth in vivo. It was demonstrated that GADD45 G induces HCC cell senescence independently of the functional presence of p16, p53 and retinoblastoma protein and that downregulation of Janus kinase (Jak)/signal transducer and activator of transcription 3 (Stat3) is the key event for GADD45G-induced cell senescence and tumor suppression. GADD45GIP1 has been demonstrated to directly bind to all GADD45 isoforms, particularly GADD45G, and the interaction between GADD45GIP1 and GADD45 members enhances GADD45 function in a cell culture system (7). Therefore, NAC1 may affect cisplatin resistance, resulting in cellular senescence through the suppression of GADD45GIP1 expression; this would inhibit Gadd $45 \mathrm{G}$ activity, thereby preventing activation of cellular senescence through the Jak and Stat 3 signaling pathways. Further studies are required to clarify the specific underlying molecular mechanisms mediating this cellular senescence cascade between GADD45GIP1/GADD45G, and the Jak or Stat 3 signaling pathway.

Biomarkers that are able to predict clinical prognosis, including treatment response and overall survival, have substantial clinical impact on the management of patients with ovarian cancer (16). To further explore the clinical relevance of NAC1/GADD45GIP1 axis alterations in ovarian carcinomas, the association between NAC1/GADD45GIP1 expression and progression-free/overall survival was 
examined in a population of patients with ovarian carcinoma in the present study. A marked association between poor prognosis and NAC1/GADD45GIP1 axis expression was identified in patients who received platinum-based chemotherapy. The underlying molecular mechanism for the association between NAC1/GADD45GIP1 axis expression and decreased survival remains unclear; however, as the mortality of patients with ovarian cancer is directly associated with recurrence of the disease following chemotherapy, it is hypothesized that expression of NAC1 and GADD45GIP1 may confer resistance to platinum-based chemotherapy and/or enhance cell proliferation in chemoresistant recurrent tumors, as demonstrated in the present study and in previous studies $(2,3,5)$.

The results of the present study suggested that targeting $\mathrm{NAC1}$ to restore the senescence response may be investigated, as a novel strategy for the treatment of platinum-resistant ovarian cancer. Furthermore, the NAC1-mediated suppression of senescence may also influence other aspects of cancer, including tumor dormancy, response to therapeutic intervention and metastasis. Exploring the effects of NAC1-mediated senescence on these features of ovarian cancer may provide insights into the importance of $\mathrm{NACl}$ and senescence in the treatment and management of platinum-resistant ovarian cancer. In addition, NAC1 has been identified to be associated with Nanog in a protein complex that is necessary for maintaining the stemness of mouse embryonic stem cells $(17,18)$. Nanog is able to prevent terminal differentiation of embryonic stem cells and sustain their pluripotency through a protein network involving NAC1 (19). The interaction between NAC1 with Nanog (19), as part of a multimember family required for maintaining the stemness of mouse embryonic stem cells, suggested that NAC1 serves a role in preventing or determining the terminal differentiation of cells. Further research is required to investigate whether this function of NAC1 in stem cells is associated with its effects on cellular senescence.

In conclusion, the results of the present study identify NAC1 as a negative regulator of cellular senescence and demonstrate that NAC1-mediated prevention of senescence, which is regulated through GADD45GIP1, serves an important role in promoting cisplatin resistance. The identification of the NAC1/GADD45GIP1 axis as a regulator of senescence, and the elucidation of the signaling pathways involved, may improve understanding of the molecular and cellular functions of this nuclear factor in ovarian cancer. Therefore, the NAC1/GADD45GIP1 axis may be a target in the treatment of ovarian cancer, particularly in the context of platinum resistance.

\section{References}

1. Siegel R, Naishadham D and Jemal A: Cancer statistics, 2012. CA Cancer J Clin 62: 10-29, 2012.

2. Ishibashi M, Nakayama K, Yeasmin S, Katagiri A, Iida K, Nakayama N, Fukumoto M and Miyazaki K: A BTB/POZ gene, NAC-1, a tumor recurrence-associated gene, as a potential target for Taxol resistance in ovarian cancer. Clin Cancer Res 14: 3149-3155, 2008.

3. Nakayama K, Nakayama N, Davidson B, Sheu JJ, Jinawath N, Santillan A, Salani R, Bristow RE, Morin PJ, Kurman RJ, et al: $\mathrm{A} \mathrm{BTB} / \mathrm{POZ}$ protein, NAC-1, is related to tumor recurrence and is essential for tumor growth and survival. Proc Natl Acad Sci USA 103: 18739-18744, 2006.
4. Jinawath N, Vasoontara C, Yap KL, Thiaville MM, Nakayama K, Wang TL and Shih IM: NAC-1, a potential stem cell pluripotency factor, contributes to paclitaxel resistance in ovarian cancer through inactivating Gadd45 pathway. Oncogene 28: 1941-1948, 2009.

5. Nakayama K, Rahman MT, Rahman M, Yeasmin S, Ishikawa M, Katagiri A, Iida K, Nakayama N and Miyazaki K: Biological role and prognostic significance of $\mathrm{NACl}$ in ovarian cancer. Gynecol Oncol 119: 469-478, 2010.

6. Nakayama K, Nakayama N, Wang TL and Shih IeM: NAC-1 controls cell growth and survival by repressing transcription of Gadd45GIP1, a candidate tumor suppressor. Cancer Res 67: 8058-8064, 2007.

7. Chung HK, Yi YW, Jung NC, Kim D, Suh JM, Kim H, Park KC, Song JH, Kim DW, Hwang ES, et al: CR6-interacting factor 1 interacts with Gadd45 family proteins and modulates the cell cycle. J Biol Chem 278: 28079-28088, 2003.

8. Zhang Y, Cheng Y, Ren X, Hori T, Huber-Keener KJ, Zhang L, Yap KL, Liu D, Shantz L, Qin ZH, et al: Dysfunction of nucleus accumbens-1 activates cellular senescence and inhibits tumor cell proliferation and oncogenesis. Cancer Res 72: 4262-4275, 2012.

9. Dimri GP, Lee X, Basile G, Acosta M, Scott G, Roskelley C, Medrano EE, Linskens M, Rubelj I, Pereira-Smith O, et al: A biomarker that identifies senescent human cells in culture and in aging skin in vivo. Proc Natl Acad Sci USA 92: 9363-9367, 1995.

10. Saretzki G: Cellular senescence in the development and treatment of cancer. Curr Pharm Des 16: 79-100, 2010.

11. Lechel A, Satyanarayana A, Ju Z, Plentz RR, Schaetzlein S, Rudolph C, Wilkens L, Wiemann SU, Saretzki G, Malek NP, et al: The cellular level of telomere dysfunction determines induction of senescence or apoptosis in vivo. EMBO Rep 6: 275-281, 2005.

12. Havelka AM, Berndtsson M, Olofsson MH, Shoshan MC and Linder S: Mechanisms of action of DNA-damaging anticancer drugs in treatment of carcinomas: Is acute apoptosis an 'off-target' effect? Mini Rev Med Chem 7: 1035-1039, 2007.

13. Odicino F, Pecorelli S, Zigliani L and Creasman WT: History of the FIGO cancer staging system. Int J Gynaecol Obstet 101: 205-210, 2008.

14. Shih IeM, Nakayama K, Wu G, Nakayama N, Zhang J and Wang TL: Amplification of the ch19p13.2 NACC1 locus in ovarian high-grade serous carcinoma. Mod Pathol 24: 638-645, 2011.

15. Zhang L, Yang Z, Ma A, Qu Y, Xia S, Xu D, Ge C, Qiu B, Xia Q, $\mathrm{Li} \mathrm{J}$ and Liu Y: Growth arrest and DNA damage $45 \mathrm{G}$ down-regulation contributes to Janus kinase/signal transducer and activator of transcription 3 activation and cellular senescence evasion in hepatocellular carcinoma. Hepatology 59: 178-189, 2014.

16. Scott $M$ and Hall PA: Prognostic and predictive factors. Methods Mol Med 97: 1-11, 2004.

17. Kim J, Chu J, Shen X, Wang J and Orkin SH: An extended transcriptional network for pluripotency of embryonic stem cells. Cell 132: 1049-1061, 2008.

18. Wang J, Rao S, Chu J, Shen X, Levasseur DN, Theunissen TW and Orkin SH: A protein interaction network for pluripotency of embryonic stem cells. Nature 444: 364-368, 2006.

19. Wang J, Levasseur DN and Orkin SH: Requirement of Nanog dimerization for stem cell self-renewal and pluripotency. Proc Natl Acad Sci USA 105: 6326-6331, 2008. 